\title{
The Expedient Approach for High Availability in Web Server Services for HPC Attained by Clustering using Virtualization
}

\author{
Mamta Gadoria \\ M.Tech CS (Student) \\ J.V.W.U \\ Jaipur
}

\author{
Sarvesh Singh \\ Assistant Professor \\ J.V.W.U \\ Jaipur
}

\author{
Pratibha Gangwar \\ M.Tech CS (Student) \\ J.V.W.U \\ Jaipur
}

\begin{abstract}
In this paper we have presented the model of clustering using Heartbeat and implemented with Virtualized environment. . For making a machine to be virtualized, the hardware must endow with virtualization capabilities we believe that we understand what makes the websites unreliable; the key to build a more reliable website is to make a cluster. Clustering is typically transparent from the clients as well as developers, perk up the system availability to the online users, as the failed server will automatically pack up and the users will automatically switched at that particular instant to the another server. Heartbeat is an open source portable tool for clustering.
\end{abstract}

\section{General Terms}

Heartbeat Apache, clustering, High availability

\section{Keywords}

HA, 2-node cluster

\section{INTRODUCTION}

Apache Heartbeat, a High availability implementation for web services, is a broadly acceptable technology. While configuring Apache Web Server, schemes have been already addressed in previous literature. Nowadays providing reliable online services is a trend for almost every organizations and enterprises. Reliability here means, reliability of the server, hosting websites. Unreliable websites are the only major source of user frustration. In 2-nodes clustering, you have one front end node and a back end node. A 2-node cluster schema is used here to make a transparent layer i.e. requirement of both application layer and transport layer mechanisms, to the clients. Although the website reliability is a key concern from the very first day it has been accessed because resubmitting the requests to the server throws bad impression to the clients. So, this reason made competition a bit higher in the field of servers. Clustering offers the finest scalability i.e. can be increased dynamically to meet the demands and high availability i.e. can persist when a server instance fails. In cluster structures, the server applications can reliably make use of the resources in the least down time. In this paper, we have spotlight on the HA clusters with heartbeat tool for hiding the server failures from the clients using clustering, an accepted technology for high availability, parallel processing applications and shared computing resources. The principle of this record is to give explanation of how to put into practice, a 2-node HA cluster. Devoid of clustering if a server running application becomes unavailable at a particular instant, then the services will not be accessible until the application server is fixed. Server cluster labels how the nodes are tied to store the cluster configurations. The impression of this approach is to supply the tightly coupled services to the clients. The model proposed here is an Active-Active Model of nodes, where both the nodes make use of homogenous software configurations. In other words we can also say that, in the event of failure on the node 1 , node 2 will automatically serve the services, i.e. restarting departed machine on the secondary node. Heartbeat program sprints on every Linux platform. High Availability scheme implemented here has a design of three Linux systems (Centos), i.e. client, primary node and secondary node. The Heartbeat tool is high performance computing tool which serves with easy observing between two systems. The resources are directed between the two nodes in the cluster, if one fails, another will serve by managing resources. By configuring the heartbeat tool for clustering user will without doubt experience a bare minimum of tumult in services that are offered by the servers. To create a cluster with 2-nodes, you need to configure the heartbeat properly with accurate hardware with network infrastructure.

\section{LITERATURE SURVEY}

Reliable online services are the most recent movement to avoid the user frustration. In this paper, we centered on the heartbeat tool for making a 2-node cluster, hiding the server failure from the clients. High availability demotes to consistency, sustainable and integrity of data access. High availability is typically capable to assemble the development in the reliability of servers and applications. Some models necessitate massive data processing in huge scale computing environments i.e. clouds. Managing a server with loosely coupled architecture is an intricate task. By creating a cluster, you can make tightly coupled environment. High availability clustering is defined by asymmetric active/active model. Both hardware and software fails, unavoidably. There can be a number of nodes which can break down the whole thing; it only necessitates the hardware to reinstate or Operating system to restart.

\section{HIGH AVAILABILITY CLUSTERING}

\subsection{Node Cluster}

The 2- node clustering through Heartbeat proffers much uncomplicated takeover semantics with dependencies and policies. Almost every protocol centered on increasing the reliability of the messages i.e. fully attentive towards the ensuring of high availability in the event. The 2-nodes in the cluster are also known as neighbors of the private network. This approach is to provide high availability to the web 
services. There are many solutions also to provide the high availability clustering, but heartbeat has a feature to support from node 2 to node $n$. The unreliability in the services is because of the primary host failure, the heartbeat automatically detects it and the released output will correspond to the secondary host. The basic need to make a High availability cluster to the web services is to configure the application properly, within the framework. To configure the 2.node cluster, you require good sound knowledge of the product and the application. Configuring the files is the difficult task to implement because there are various steps to go through to configure it properly, since the configuration setup is different for different applications. Even easy websites also need to be configuring accurately within the cluster system to impart high availability. . Majority of the failures of websites, on high availability cluster is due to the configuration files on the clustering environment made by the people. The advantage of clustering $\mathrm{HA}$ is that the configuration files are totally concealed from clients as well as developers. HA made application to budge from one compute failure node to another compute node, also termed as failover.

\subsection{Heartbeat Apache}

We have implemented Heartbeat high availability with Apache Web Server. The daemon that presents communication and membership i.e. cluster infrastructure services private network to clients, which consents reliable exchange of messages. Heartbeat, on the whole creates a private network, shared by the 2-nodes of cluster, where no other node can have right to admission in this private network. This is the property of heartbeat that events in heartbeat are appropriately ordered according to the FIFO nature of signals, making confident that all the messages have been arriving properly. The most vital attribute of heartbeat, which makes it highly available, is that the resources have been automatically, transparently transfers to the node, when one node fails. It offers monitoring and controlling of resources through configuring the files in the nodes. Heartbeat has become one of the well-liked frameworks to process the data on the cluster. Due to the attractiveness of HPC, heartbeat gains the researchers attention. This tool has the property to perceive fast that which node is not working appropriately, to transfer the load to another node. Heartbeat is totally accountable to serve the services to certify client requests, according to the failure of node1 or failure of node 2, because the unavailability of services leaves unenthusiastic impact to the clients. There may be certain events that can quickly augment the significant load that persevere for hours, throws bad impression to the clients.

\section{DESIGN AND IMPLEMENTATIONS}

To implement the Heartbeat, the following steps need to be taken:

A. You need to install two Linux machines to make two nodes ( we have used Centos, you can use any flavor of Linux), and a client machine

B. You need to make a Yum Server and client.

C. Then, make a DNS server and make its client.

D. After configuring your DNS server, make Apache Web Server and configure it.

E. Then, in the last step, you need to install Heartbeat packages and configure Heartbeat i.e. you need to make changes to the three files of Heartbeat i.e. /etc/ha.d/authkeys, /etc/ha.d/haresources, /etc/ha.d/ha.cf on node 1 and then you need to copy these files to the node 2 .

You can assign the IP address we have implemented; we have used the concept of virtualization as we have implemented it on the single machine. Shown in the fig 1

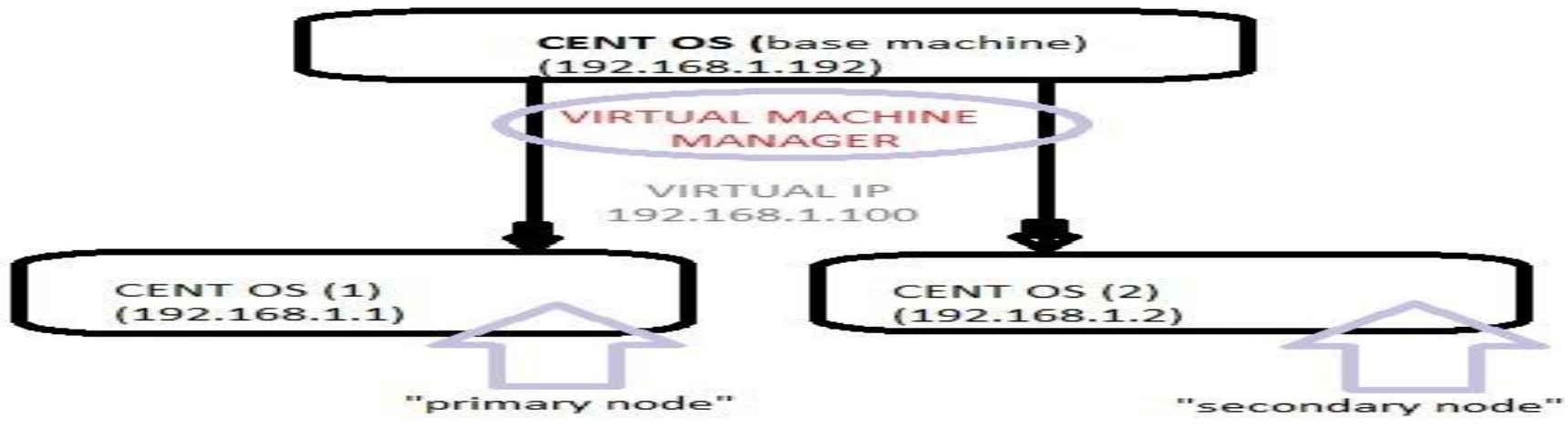

Fig 1. Showing the design of implementation

\section{RESULTS}

For assigning virtual ip address, eth0:0 must be configured, as shown in fig 2 . 

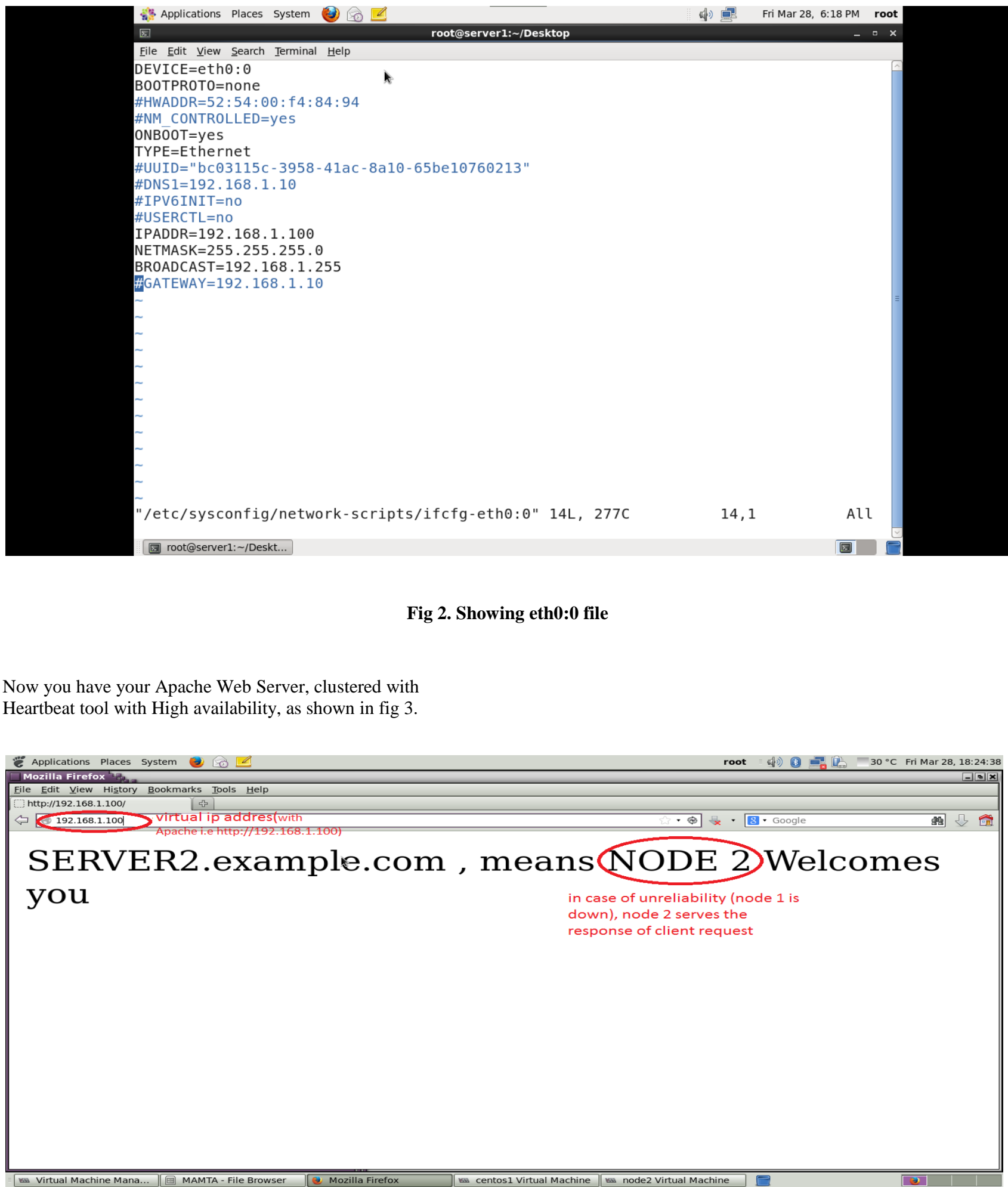

Fig 3. Failure of node 1, node 2 is running

\section{PERFORMANCE}

Cluster architecture supports HA, which is gradually more being vision as a successful and price effective approach for the HPC. In this approach, a reliable, scalable and high performatic Apache made through Heartbeat, to access the services of internet. Cluster services principally accommodate the enlargement of web traffic. . This technique serves compatibility (no adaptation from the client side), small price (no devoted hardware), and little overhead (no extra delay). Serviceability which is also known as RaS taxonomy.

\section{FUTURE WORK AND CONCLUSION}

The implementations and study will be very supportive for the researchers of High availability in future. We have implemented and worked with Apache Web Server, but it can also be implemented with Mail server, Ftp, Samba also. Heartbeat is the absolute solution that provides you high 
availability with high performance. Our work is to grant the reliable access to the web services, implementations are correct and offer better RAS.

\section{REFERENCES}

[1] Ronghua Zhang, Tarek F. Abdelzaher, John A. Stankovic. Efficient TCP Connection Failover in Web Server Clusters. 0-7803-8356-7/04/\$20.00 (C) 2004 IEEE.

[2] Darys Chudova ,Scott Gaffney ,and Padgrai Smyth. Probabilistic Models for Joint Clustering and TimeWarping of Multidimensional Curves 134, CHUDOVA ET AL UAI2003.

[3] Benjamin Satzger ,Andreas Pietzwski , Woldgang trumler , and Theo Ungerer. A Lazy monitoring for Heartbeat-Style Failure Detectors .0-7695-3102- 4/08 2008 IEEE DOI 10.1109/ARES.2008.51.

[4] C. Engelmann1;2, S. L. Scott1, C. Leangsuksun3, X. Hey Symmetric Active/Active High Availability for HighPerformance Computing System Services: Accomplishments and Limitations. 978-0-7695-31564/08 \$25.00 @ 2008 IEEE.

[5] Qun Li, Ligang Dong and Ming Gao. Research on HighAvailability Based on Architecture of ForCES. 978-07695-3699-6/09 \$25.00 @ 2009 IEEE.

[6] Koichi Shirahata*, Hitoshi Sato* and Satoshi Matsuoka. Hybrid. Map Task Scheduling for GPU-based Heterogeneous Clusters. 978-0-7695-4302-4/10 \$26.00 (c) 2010 IEEE.
[7] Shantanu Sharma and Awadhesh Kumar Singh. Democratic Leader Finding Algorithm for Large Mobile Ad Hoc Networks. 1545-0678/11 \$26.00 @ 2011 IEEE.

[8] Adrian Coles, Ioan Stan. Improving the Responsiveness of Replicated Virtualized Services in Case of Overloaded Replicas Connectivity. 978-0-7695-4934-7/12 \$26.00 (c) 2012 IEEE.

[9] Augustus F Diraviam, Ritu Agrawal, Madhur Bansal, Krishna Janakiraman. CHAF - An Object-Oriented Framework for Configuring Applications in a Clustered Environment. 1-4244-1388-5/07/\$25.00 @ 2007 IEEE.

[10] Peter Bull, Lin Guan, Iain Phillips. A quality of service framework for Dependability in Large scale distributed systems. 978-1-4673-0412-2/11/\$26.00 (02011 IEEE.

[11] Eltefaat Shokri, Periklis Beltas. An Experiment with Adaptive Fault Tolerance in Highly-Constraint Systems.

[12] Chu-Sing Yang, Mon-Yen Luo. Building of Adaptable, fault tolerant and highly available clusters of Nondedicated Workstations. 2000 International Conference on Parallel Processing (ICPP'00)0-7695-0768-9/00 \$10.00@ 2000 IEEE.

[13] I.Ling Yen, Member, IEEE, Farokh B. Bastani, Member, IEEE, and David J. Taylor, Member, IEEE. Design of Multi-Invariant Data Structure for Robust Shared Accesses.

[14] In Multiprocessor Systems. Ieee Transactions on Software Engineering, Vol. 27, no. 3,March 2001. 